\title{
The Influence of Electric Field on the Optical Spin Polarization of Electrons in a Diluted Magnetic Semiconductor
}

\author{
P.M. Gorley ${ }^{a}$, O.M. Mysliuk ${ }^{a}$, V.K. DugaeV ${ }^{b, c}$, P.P. Horley ${ }^{d, a}$ \\ AND J. BARNAŚ ${ }^{e}$ \\ ${ }^{a}$ Chernivtsi National University, 2 Kotsyubynsky Str., 58012 Chernivtsi, Ukraine \\ ${ }^{b}$ University of Technology, al. Powstańców Warszawy 6, 35-959 Rzeszów, Poland \\ ${ }^{c}$ Instituto Superior Técnico, Av. Rovisco Pais, 1049-001 Lisbon, Portugal \\ ${ }^{d}$ Centro de Investigación en Materiales Avanzados, S.C., Chihuahua/Monterrey \\ Av. Miguel de Cervantes 120, 31-109 Chihuahua, Chih., México \\ ${ }^{e}$ A. Mickiewicz University, Umultowska 85, 61-614 Poznań, Poland
}

\begin{abstract}
In this paper we present the results of theoretical calculations for spin polarization $\eta$ of band electrons in diluted magnetic semiconductor subjected to a polarized light wave and a carrier-warming electric field $E$. It was shown that the maximum value of $\eta_{\max }$ can be reached at a certain $E_{\max }$ corresponding to the peak of the carrier drift velocity $v(E)$. For the higher doping impurity concentration, the values of $\eta_{\max }$ become lower due to the equivalent decrease of electron temperature.
\end{abstract}

PACS numbers: 72.25.Fe, 72.25.Rb, 72.25.-b

\section{Introduction}

Optical spin polarization of electrons in diluted magnetic semiconductors (DMS) attracted significant scientific attention, opening the perspectives for the development of efficient spintronic devices (e.g. [1] and the references therein). Our previous research $[2,3]$ revealed that high spin polarization $\eta$ of the conduction band electrons in DMS can be achieved by subjecting the sample to a weak magnetic field together with a circularly-polarized light wave. In this paper we present the results of theoretical analysis concerning the joint action of carrier-warming electric field and a polarized light wave on the magnitude of carrier spin polarization degree. The calculations were performed for the model semiconductor $n$-InP:F using band parameters from Ref. [3].

\section{Theory}

We considered a diluted magnetic semiconductor with shallow impurity levels (donors and acceptors with concentrations $N_{\mathrm{d}}$ and $N_{\mathrm{a}}$, respectively), completely ionized at the room temperature and thus determining the $n$-type conductivity of the material. Additionally, we assume the presence of a deep level formed by the element with non-filled $d$ - or $f$ - shell with the concentration $N_{\text {T }}$. The frequency of an incident circularly polarized light wave was supposed to satisfy impurity absorption condition. The kinetics of optical transitions in this case can be described with non-linear differential equations [2]. To expand the approach used in [2], we also took into account the non-linear field dependence of electron drift velocity $v(E)$. Upon this general treatment of the problem, the resulting equations become complicated and cannot be presented in full form in this paper. The equations were solved with 4th order Runge-Kutta method using InP:Fe parameters from Ref. [4], $T=300 \mathrm{~K}$. The experimental $v(E)$ curves measured for different impurity concentration [5] were approximated with an empirical formula

$$
\nu(E)=\nu_{S} \frac{E / E_{S}+A\left(E / E_{S}\right)^{3}}{1+B\left(E / E_{S}\right)^{3}}
$$

with dimensionless coefficients $A$ and $B$. The latter are equal to 0.047 and 0.22 , respectively, for the doping impurity concentration $N_{\mathrm{T}}=10^{20} \mathrm{~m}^{-3}$; for $N_{\mathrm{T}}=10^{21} \mathrm{~m}^{-3}$ one will obtain $A=0.119$ and $B=0.27$. The other values used in (1) are $v_{S}=2.7 \times 10^{5} \mathrm{~m} / \mathrm{s}$ and $E_{S}=$ $0.8 \times 10^{6} \mathrm{~V} / \mathrm{m}[4,5]$. The calculated $v(E)$ are given in Fig. 1 as solid curves; the experimental data [5] are shown with circles.

\section{Results and discussion}

The results of numerical calculations for impurity concentration $N_{\mathrm{T}}=10^{20} \mathrm{~m}^{-3}$ reveals that for all types of 


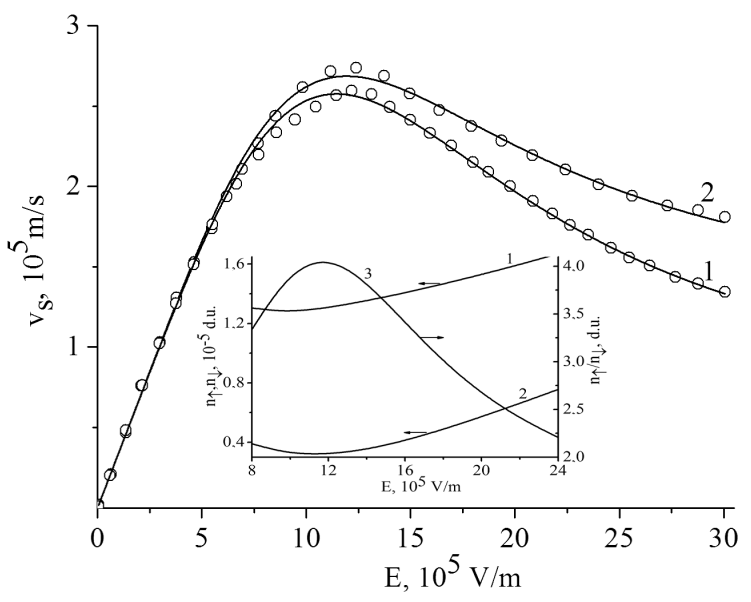

Fig. 1. The $v(E)$ plots: circles - experimental data [5]; solid curves - calculation results obtained with formula (1). Impurity concentration $N_{\mathrm{T}}$ : (1) $N_{\mathrm{T}}=10^{20} \mathrm{~m}^{-3}$, (2) $N_{\mathrm{T}}=10^{21} \mathrm{~m}^{-3}$. The inset shows $n_{\uparrow}, n_{\downarrow}$ and ratio thereof (curves $1-3$, respectively) as a function of $E$.

light wave polarization the curves $\eta(E)$ are characterized with a peak, which is highest $\left(\eta_{\max } \approx 60 \%\right.$ for InP) when the incident light has left-hand polarization. The magnitude of $\eta$ in DMS subjected to carrier-warming fields depends significantly on the spin relaxation times both in sub-system of magnetic ions and conduction band electrons.

The inset to Fig. 1 presents the calculated band electron concentrations for the carriers with spins up $n_{\uparrow}$ and down $n_{\downarrow}$ (curves 1 and 2, respectively) and their ratio $n_{\uparrow} / n_{\downarrow}$ as curve 3 .

As one can see, under electric fields causing deviation from Ohm's law and up to the point $E_{\max }$ corresponding to the peak of $v(E)$ curve, both $n_{\uparrow, \downarrow}$ decrease with an increasing field. The slope for the concentration $n_{\downarrow}$ is steeper than that of $n_{\uparrow}$. In the region of negative differential resistivity the increase of $E$ increases the concentrations $n_{\uparrow, \downarrow}$, with $n_{\downarrow}$ growing faster than $n_{\uparrow}$. These peculiarities of $n_{\uparrow, \downarrow}=f_{\uparrow, \downarrow}(E)$ are reflected in the behavior of their ratio $\eta_{\mathrm{e}}=\left(n_{\uparrow}-n_{\downarrow}\right) /\left(n_{\uparrow}+n_{\downarrow}\right)$ as a function of $E$, which features a distinctive peak.

For the left-handed polarization of the incident light the increase of $N_{\mathrm{T}}$ from $10^{20} \mathrm{~m}^{-3}$ to $10^{21} \mathrm{~m}^{-3}$ (Fig. 2, curves 1 and 2) the maximum $\eta$ decreases by $8 \%$, which is caused by practically equivalent decrease of electron temperature due to the higher electron concentration. For $N_{\mathrm{T}}=10^{21} \mathrm{~m}^{-3}$, the slope of $\eta_{\mathrm{e}}=f\left(E / E_{S}\right)$ plot becomes lower and the general shape of the curve quantitatively agrees with the experimental data for GaAs [6] regarding the photoluminescence polarization under the warming electric fields and interband absorption conditions (inset to Fig. 2). These results suggest the existence of an optimal impurity concentration $N_{\mathrm{T}}$ that will allow to obtain the maximum possible $\eta$ for the given DMS subjected to a polarized light-wave and electric field.

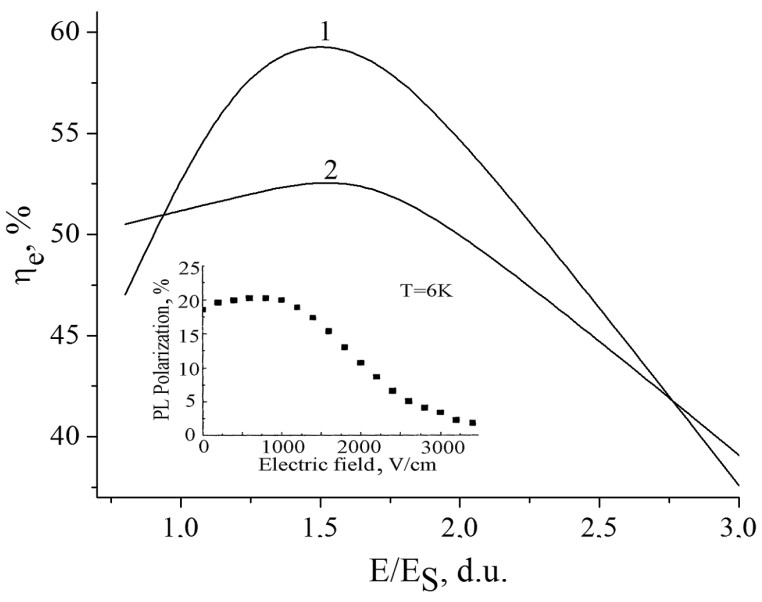

Fig. 2. $\eta_{\mathrm{e}}\left(E / E_{S}\right)$ curves for illumination with left-handed polarized light. Impurity concentration: (1) $N_{\mathrm{T}}=10^{20} \mathrm{~m}^{-3},(2) N_{\mathrm{T}}=10^{21} \mathrm{~m}^{-3}$. The inset shows the experimental data for $\eta$ obtained from the photoluminescence measurements in GaAs [6].

\section{Conclusions}

We report significant increase of spin polarization degree $\eta$ varying from $47 \%$ in weak fields to $\approx 60 \%$ in carrier-warming fields for InP:Fe semiconductor under the simultaneous action of left-hand polarized light and electric field. The predicted effect is caused by peculiar field dependence of carrier concentration profiles $n_{\uparrow, \downarrow}(E)$. The increase of impurity concentration $N_{\mathrm{T}}$ decreases the maximum spin polarization $\eta_{\mathrm{e} \text { max }}$ due to an equivalent lowering of electron temperature.

\section{Acknowledgments}

This work was partially supported by the budget costs of the Ukrainian Ministry of Education and Science assigned for financing of research projects carried out at the Department of Electronics and Energy Engineering in 2009-2010.

\section{References}

[1] I. Zutić, S. Fabian, S. Das Sarma, Rev. Mod. Phys. 76, 323 (2004).

[2] P.M. Gorley, V.K. Dugaev, J. Barnas, P.P. Horley, O.M. Mysliuk, J. Phys., Condens. Matter 19, 266205 (2007).

[3] P.M. Gorley, O.M. Mysliuk, M. Vieira, P.P. Horley, V.K. Dugaev, J. Barnas, Ukr. J. Phys. Opt. 9, 62 (2008).

[4] K. Pressel, G. Bohnert, A. Dorner, B. Kaufmann, Phys. Rev. B 147, 9411 (1993).

[5] T.J. Maloney, J. Prey, J. Appl. Phys. 48, 781 (1977).

[6] H. Sanada, I. Arata, Y. Ohno, K. Ohtani, Z. Chen, K. Kayanuma, Y. Oka, F. Matsukura, H. Ohno, J. Superconductivity 16, 217 (2003). 